\title{
A qualidade da dieta de usuários de serviço de promoção da saúde difere segundo o comportamento alimentar obtido pelo modelo transteórico ${ }^{1}$
}

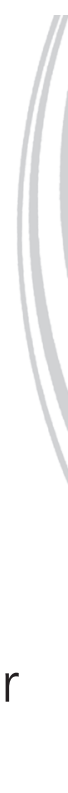

ORIGINAL | ORIGINAL

\author{
Diet quality of health-promoting services \\ users and eating behavior according \\ to the transtheoretical model
}

Renata Andrade de Medeiros MOREIRA²

Luana Caroline dos SANTOS 3

Aline Cristine Souza LOPES 3

\section{RE S U M O}

\section{Objetivo}

Investigar a qualidade da dieta de acordo com o comportamento alimentar para consumo de óleos e gorduras de usuários de serviço de saúde.

\section{Métodos}

Estudo seccional com usuários de Serviço de Promoção da Saúde. A qualidade da dieta foi avaliada pelo teste "Como está sua alimentação?", e o comportamento alimentar pelo Modelo Transteórico para consumo de óleos e gorduras, reclassificado pela ingestão de lipídeos totais.

\section{Resultados}

Dos 145 avaliados, 49,6\% foram classificados no grupo pré-ação (estágios de pré-contemplação, contemplação e decisão). Esses usuários apresentaram maior consumo de alimentos ricos em gordura e menos saudáveis, e, consequentemente, maiores médias de consumo de calorias, lipídeos, ácidos graxos saturados e colesterol, e menores de carboidratos e ácidos graxos monoinsaturados. Além disso, apresentaram maior prevalência de dieta inadequada: $76,4 \%$ dos indivíduos que estavam em pré-ação vs. $67,1 \%$ daqueles em ação deviam ficar atentos com a alimentação $(p=0,02)$.

\footnotetext{
1 Apoio: Fundação de Amparo à Pesquisa do Estado de Minas Gerais (Processo no 13201).

2 Pontifícia Universidade Católica de Minas Gerais, Curso de Nutrição. Barreiro, MG, Brasil.

${ }^{3}$ Universidade Federal de Minas Gerais, Escola de Enfermagem, Departamento de Enfermagem Materno-Infantil e Saúde Pública, Grupos de Pesquisas de Intervenções em Nutrição. Av. Alfredo Balena, 190, 4º andar, Sala 420, Santa Efigênia, 30130-100, Belo Horizonte, MG, Brasil. Correspondência para/Correspondence to: ACS LOPES. E-mail: <aline@enf.ufmg.br>.
} 
720 | RAM MOREIRA et al.

\section{Conclusão}

A similaridade dos resultados obtidos a partir dos métodos evidencia a necessidade de se avaliar os hábitos alimentares, em conjunto, visando identificar a necessidade de mudanças desses hábitos, bem como contribuir para o melhor delineamento, implementação e avaliação de intervenções, de acordo com os estágios de mudança comportamental.

Termos de indexação: Comportamento alimentar. Consumo alimentar. Hábitos alimentares. Recomendações nutricionais.

\section{A B S T R A C T}

\section{Objective}

This study investigated the dietary quality of users of a healthcare service and their eating behavior with respect to oils and fats.

\section{Methods}

This was a cross-sectional study with users of a health-promoting service. Dietary quality was assessed by the instrument "How is your diet" and eating behavior by the transtheoretical model for the consumption of oils and fats, reclassified as total fat intake.

\section{Results}

Among the 145 respondents, 49.6\% were classified as being in the pre-action group (stages of precontemplation, contemplation and decision). These users consumed more high-fat foods and fewer healthy foods, therefore, their mean intakes of calories, fat, saturated fats and cholesterol were higher, and mean intakes of carbohydrate and monounsaturated fats were lower. Furthermore, they were more likely to consume inappropriate diets (according to the test, $76.4 \%$ should pay attention to their diet vs. $67.1 \%$ of users in the action stage, $p=0.02$ ).

\section{Conclusion}

The similarity of the data obtained by the methods highlights the need of assessing dietary habits and eating behavior and identifies the need of changing dietary habits and better planning, implementing and assessing interventions, according to behavioral change stages.

Indexing terms: Feeding behavior. Food consumption. Food habits. Nutritional recommendation.

\section{N T R O D U ÇÃ O}

As mudanças dietéticas ocorridas na população brasileira nos últimos anos propiciaram novos padrões de nutrição e favoreceram o aumento da obesidade. Dentre as mudanças alimentares, destacam-se o aumento da ingestão de alimentos ricos em gorduras, principalmente de origem animal, e de açúcar, bem como a maior frequência de consumo de alimentos industrializados e de refeições realizadas fora de casa, e a redução da ingestão de carboidratos complexos, leguminosas, frutas, verduras e legumes na dieta

Na comparação entre as Pesquisas de Orçamentos Familiares (POF) de 2002/2003 e 2008/2009, verificou-se aumento do consumo de lipídeos (27,8\% para 28,7\%), proveniente da maior ingestão de ácidos graxos monoinsaturados $(8,7 \%$ para $9,2 \%$ ) e saturados (7,9\% para $8,3 \%)$, e do aporte proteico (11,6\% para $12,1 \%)$, sendo os alimentos de origem animal os responsáveis por esse aumento (6,1\% para 60,7\%). Apesar da estabilidade da participação dos açúcares livres na dieta em $16,4 \%$, ressalta-se que o limite máximo de 10,0\% para a proporção de calorias provenientes desse nutriente, assim como de lipídeo total e de ácidos graxos saturados, foi ultrapassado, revelando um padrão alimentar pouco saudável².

Corroborando esses dados, na pesquisa Vigilância de Fatores de Risco e Proteção para Doenças Crônicas por Inquérito Telefônico, obser- 
vou-se que a população adulta brasileira, em 2011, apresentou baixo (30,9\%) consumo regular de frutas e hortaliças ( $\geq 5$ porções/semana) - apenas $20,2 \%$ consumia o número de porções recomendadas ( $\geq 5$ porções/dia) -, além de redução da ingestão regular de feijão ( $\geq 5$ dias da semana) para 69,1\%. Quanto à ingestão e alimentos que constituem fator de risco à saúde, constatou-se que $34,6 \%$ ingerem carne com excesso de gordura, $56,9 \%$ leite integral e $29,8 \%$ consomem refrigerantes regularmente (5 vezes/semana) ${ }^{3}$.

Essa maior disponibilidade de alimentos com alta densidade calórica e pobres em micronutrientes aumenta o risco de doenças, em especial as Doenças e Agravos Não-Transmissíveis (DANT) $)^{1,2-4}$ como obesidade, diabetes e cânceres, e contribui substancialmente para a carga mundial de morbidade, mortalidade e incapacidade ${ }^{1}$.

Segundo a Organização Mundial de Saúde (OMS), o consumo insuficiente de frutas e hortaliças (per capita <400g ou 5 porções diárias) é responsável por 2,7 milhões de mortes e 31\% das doenças isquêmicas do coração, $11 \%$ das doenças cerebrovasculares, e 19\% dos cânceres gastrointestinais no mundo. Além disso, as dislipidemias são causadas, em sua maioria, pelo elevado consumo de gordura saturada de origem animal, causando 4,4 milhões de mortes, 18\% das doenças cerebrovasculares e $56 \%$ das doenças isquêmicas do coração ${ }^{4}$.

Assim, a avaliação dos hábitos alimentares apresenta-se cada vez mais em evidência devido sua estreita relação com a prevenção e o controle de doenças, e a promoção da saúde ${ }^{1,5}$. Sua importância também se traduz por possibilitar identificar indivíduos e grupos em risco nutricional; facilitar o planejamento, monitoramento e avaliação de programas de intervenção; e subsidiar políticas nacionais de saúde e nutrição ${ }^{1,5}$. Apesar de sua importância, a avaliação do consumo de alimentos ainda constitui um desafio, principalmente no que se refere à mensuração da alimentação saudável e do comportamento alimentar.
Nesse sentido, o Ministério da Saúde propôs um instrumento para analisar a qualidade da dieta de adultos e idosos e grupos populacionais: o teste "Como está sua alimentação?", que propõe realizar a avaliação global da dieta de forma simplificada, mediante o questionamento sobre o consumo de gorduras total e saturada; colesterol; grupos das frutas, hortaliças e cereais, dentre outros ${ }^{6}$.

O comportamento alimentar é outro importante aspecto atualmente estudado por se relacionar estreitamente ao padrão alimentar adotado pelo indivíduo ou grupo. Sua análise tem sido realizada mediante o uso de modelos teóricos, composto por várias teorias, com destaque para o Modelo Transteórico ${ }^{7}$, considerado um instrumento promissor de auxílio à compreensão da mudança comportamental, sugerindo que quando os indivíduos realizam mudanças no comportamento, eles as fazem em estágios, possibilitando a reflexão sobre o comportamento, a atitude a se tomar e o momento de agir8.

Para fins de análise, os estágios podem ser alocados em dois grupos com características distintas, pré-ação e ação. No grupo pré-ação, estão incluídos os indivíduos nos estágios de pré-contemplação, contemplação e decisão, caracterizados pela não adoção de mudanças no comportamento no momento. Os indivíduos pertencentes ao grupo ação são aqueles que estão nos estágios de ação e manutenção, ou seja, efetivamente realizam alterações de maneira consistente em seu comportamento, exigindo dedicação e disposição para evitar recaídas? ${ }^{7}$.

O confronto entre a qualidade da dieta e o comportamento alimentar possibilita conhecer o significado simbólico dos alimentos para os sujeitos, visando construir novos sentidos para o ato de comer ${ }^{9}$. Assim, considerando a importância de se conhecerem esses aspectos relacionados à alimentação, este estudo objetivou investigar a qualidade da dieta de acordo com o comportamento alimentar, pautado no modelo transteórico, de usuários de Serviço de Promoção da Saúde. 


\section{MÉTODOS}

O estudo foi desenvolvido em Serviço Público de Promoção da Saúde denominado Academia da Cidade, localizado em Belo Horizonte (MG), em uma das áreas de maior vulnerabilidade social da cidade. Trata-se de um serviço do Sistema Único de Saúde que objetiva fornecer a prática regular de exercício físico e acompanhamento nutricional aos usuários, visando construir uma cidade mais saudável ${ }^{10}$

Realizou-se estudo transversal com uma amostra aleatória simples representativa dos usuários com 20 ou mais anos do serviço em questão. Os parâmetros amostrais utilizados para fins descritivos foram nível de significância de 5\%, poder de explicação de $80 \%$ da população assídua da academia no período de outubro de 2007 a novembro de 2008 ( $n=336)$, obtendo-se uma amostra de 168 indivíduos.

Com a finalidade de proporcionar homogeneidade de participação das turmas do serviço, os usuários foram sorteados aleatoriamente, sendo $50 \%$ para a turma 1 de prática de atividade física (segundas, quartas e sextas); e 50\% para a turma 2 (terças, quintas e sábados).

O instrumento de coleta de dados constou de dados sociodemográficos, antropométricos, avaliação da qualidade da dieta por meio do teste "Como está sua alimentação?"6, ingestão mensal de óleo per capita, aplicação de três Recordatórios Alimentares de 24 horas (R24) e algoritmo para consumo de óleos e gorduras ${ }^{8}$ devidamente validado para a população em questão ${ }^{11}$.

As medidas antropométricas de peso, altura, Circunferências da Cintura (CC) e do Quadril (CQ) foram aferidas de acordo com as recomendações da OMS ${ }^{12}$, e propiciaram o cálculo do Índice de Massa Corporal $(\mathrm{IMC}=$ peso/altura²) e da razão cintura/quadril (RCQ= CC/CQ). O IMC foi classificado segundo o critério preconizado pela OMS $^{12}$ para adultos e conforme a recomendação do Nutrition Screening Iniative ${ }^{13}$ para idosos. A partir da CC e da RCQ, foram avaliados os riscos de complicações metabólicas e para o desenvolvi- mento de doenças, respectivamente, de acordo com o preconizado pela $\mathrm{OMS}^{14}$.

O teste "Como está sua alimentação?"6 avalia as porções do grupo das frutas, hortaliças e cereais, carne, leguminosas e leite, alimentos ricos em gorduras e açúcares; tipo de gordura utilizada para cozinhar; consumo de gordura saturada e colesterol, e sal de adição; número de refeições; ingestão de água e de bebidas alcoólicas; o uso de informações nutricionais dos rótulos de alimentos como orientação para alimentação saudável; e prática de atividade física.

O algoritmo utilizado para identificação dos estágios de mudança referiu-se ao consumo de óleos e gorduras, proposto por Greene \& Rossi ${ }^{8}$. Na primeira parte desse instrumento, o indivíduo relata sua percepção quanto ao consumo de óleos e alimentos ricos em gorduras, classificando-se em um dos cinco estágios de mudança de comportamento alimentar. A segunda parte compreende a reclassificação dos indivíduos que se classificaram em ação e manutenção, e é realizada mediante a média da ingestão de lipídeos de três R24. Os indivíduos que relataram consumo de lipídeos igual ou inferior a 30\% são mantidos nos estágio de mudança de ação e manutenção, enquanto os demais (ingestão de gorduras superior a 30\%) são reclassificados para os estágios de pré-contemplação, contemplação e decisão, mediante quatro perguntas relativas ao consumo de alimentos ricos em gordura e uma relacionada ao consumo de frutas, legumes e verduras em lanches com alimentos gordurosos 8,11 .

Os recordatórios alimentares de 24 horas foram aplicados por entrevistadores devidamente treinados, incluindo bolsistas de iniciação científica e mestrandos envolvidos no estudo, sendo a aplicação realizada em dias alternados, abrangendo dois dias durante a semana e um de final de semana. Também foi utilizado um kit com as medidas caseiras mais utilizadas pelos usuários com o objetivo de minimizar as dificuldades em estimar porções ${ }^{15}$.

A ingestão mensal de óleo per capita foi obtida pelo autorrelato da quantidade de latas 
de óleo utilizada na residência do indivíduo em um mês, sendo esse valor dividido pelo número de pessoas que utilizavam esse produto na casa. A adequação desse consumo foi realizada considerando a recomendação do Guia Alimentar para a População Brasileira, que orienta que o consumo de óleo não deve exceder $240 \mathrm{~mL} /$ mês, per capita ${ }^{5}$.

Para a análise, os alimentos consumidos foram convertidos em gramas e transformados em nutrientes pelo programa DietWin, software de Nutrição e por diferentes tabelas de composição de alimentos, e por preparações e rótulos de alimentos industrializados quando necessário.

A avaliação do consumo de calorias e de macronutrientes foi realizada pela média dos três R24, de acordo com o Institute of Medicine ${ }^{16}$, e de ácidos graxos e colesterol segundo a OMS ${ }^{17}$.

Para análise dos dados, os indivíduos foram agrupados em pré-ação (pré-contemplação, contemplação e decisão) e ação (ação e manutenção), de acordo com os estágios de mudança do comportamento alimentar para o consumo de óleos e gorduras.

Realizou-se análise descritiva dos dados, sendo aplicado o teste Kolmogorov-Smirnov para avaliar a distribuição das variáveis. Os resultados foram apresentados sob a forma de média e desvio-padrão para as variáveis com distribuição normal e mediana e Intervalo de Confiança de 95\% para as demais. Foram aplicados os testes $t$ de Student, exato de Fisher, Mann-Whitney e Qui-quadrado de Pearson, adotando-se nível de significância de 5\%.

O estudo foi aprovado pelos Comitês de Ética da Universidade Federal de Minas Gerais (COEP n ${ }^{\circ}$ ETIC 103/07) e da Pre-feitura Municipal de Belo Horizonte (COEP - SMSA/PBH - Protocolo n 087/2007). Os usuários, informados sobre os objetivos e métodos do estudo, assinaram o Termo de Consentimento Livre e Esclarecido.

\section{RES U LTA D OS}

Dos 168 usuários selecionados, houve uma perda de $13,7 \%$ devido a recusas $(n=18)$ ou ao não preenchimento de todos os três R24 $(n=5)$, sendo avaliados 145 indivíduos. Destes, 91,0\% eram do sexo feminino, com média de idade de 53,4, Desvio-Padrão (DP) =12,3 anos, apresentando baixa renda e escolaridade. As prevalências de excesso de peso $(63,4 \%)$ e de riscos de complicações metabólicas e para o desenvolvimento de doenças, mensuradas pela CC e RCQ $(69,7 \%$ e $66,9 \%$, respectivamente), foram igualmente elevadas. As demais informações sociodemográficas e referentes ao estado nutricional da amostra estão na Tabela 1.

A classificação dos estágios de mudança do comportamento alimentar para consumo de óleos e gorduras revelou que 5,5\% dos usuários apresentavam-se em pré-contemplação, 20,0\% em contemplação, 24,1\% em decisão, 29,0\% em ação e $21,4 \%$ em manutenção. Dessa forma, 49,6\% foram agrupados no grupo Pré-Ação (PA) e 50,4\% no grupo Ação (A).

Detectou-se que as ingestões de calorias (PA:1882,24, DP $=542,4 \mathrm{kcal}$ vs. A:1636,04, $\mathrm{DP}=548,0 \mathrm{kcal} ; p=0,017)$, de lipídeos (PA:34,74, $\mathrm{DP}=4,9 \%$ vs. $\mathrm{A}: 29,64, \mathrm{DP}=5,4 \% ; p<0,001)$, de Ácido Graxo Saturado (AGS - PA:10,1\% vs. A: $8,1 \% ; p<0,001)$ e de colesterol (PA:200,8mg, 4mg vs. $A: 136,6 \mathrm{mg} ; p=0,004)$ foram superiores entre os indivíduos em pré-ação, enquanto o consumo de carboidratos (PA: 49,54, DP=6,5\% vs. A: 54,4, $\mathrm{DP}=6,7 \%, p<0,001)$ foi inferior nesse mesmo grupo (Tabela 2).

Notou-se também uma pior adequação qualitativa dos nutrientes entre os indivíduos em pré-ação, apresentando menor consumo adequado de carboidratos (PA:80,6\% vs. A:87,7\%; $p=0,0034$ ); lipídeos (PA:66,7\% vs. A:89,0\%; $p<0,001$ ), Ácido Graxo Saturado (AGS - PA:52,8\% vs. $A: 80,8 \% ; p<0,001)$ e Ácido Graxo Poli-insaturado (AGP - PA: $47,2 \%$ vs. A: $67,1 \% ; p=0,047$ ). Além disso, as ingestões excessivas de lipídeos (PA:33,3\% vs. A: $8,2 \% ; p<0,001$ ), de AGS (PA:47,2\% vs. A: $19,2 \% ; p<0,001)$ e de AGP (PA:44,4\% vs. A:26,0\%; $p=0,047)$ foram superiores nesse grupo (Tabela 2 ). 
724 | RAM MOREIRA et al.

Tabela 1. Caracterização da amostra quanto às características sociodemográficas e antropometria. Belo Horizonte (MG), 2008-2009.

\begin{tabular}{|c|c|c|}
\hline Variáveis & & Valores \\
\hline Dados Sociodemográficos & & - \\
\hline Sexo feminino (\%) & 91,00 & (IC95\%: 89,8-92,2) \\
\hline Idade (anos) & \multicolumn{2}{|c|}{$M=53,4, I D P=12,3$} \\
\hline Faixa etária (\%) & & - \\
\hline Adultos & 66,90 & (IC95\%: 63,3-70,5) \\
\hline Idosos & 33,10 & (IC95\%: 27,93-38,27) \\
\hline Estudo (anos) & 6,50 & (IC95\%: 6,1-7,7) \\
\hline Renda mensal per capita (dólares) & 194,15 & (IC95\%: 203,42-264,47) \\
\hline Ocupação profissional (\%) & & - \\
\hline Do lar & 42,60 & (IC95\%: 37,6-47,6) \\
\hline Aposentado & 19,70 & (IC95\%: 14,9-24,5) \\
\hline Artesão & 3,30 & (IC95\%: 0,9-5,7) \\
\hline Doméstica & 3,30 & (IC95\%: 0,9-5,7) \\
\hline Desempregado & 2,50 & (IC95\%: 0,4-4,6) \\
\hline Outras & 28,60 & (IC95\%: 23,5-33,7) \\
\hline Antropometria & & - \\
\hline Estado nutricional (\%) & & - \\
\hline Baixo peso & 2,10 & (IC95\%: 0,2-4,0) \\
\hline Eutrofia & 34,50 & (IC95\%: 29,3-39,7) \\
\hline Excesso de peso & 63,40 & (IC95\%: 59,5-67,3) \\
\hline Risco de complicações metabólicas (\%) & & - \\
\hline Sem risco & 30,30 & (IC95\%: 25,1-35,5) \\
\hline Risco elevado & 22,80 & (IC95\%: 17,8-27,75) \\
\hline Risco muito elevado & 46,90 & (IC95\%: 42,0-51,8) \\
\hline Risco para desenvolver de doenças (\%) & & - \\
\hline Sem risco & 33,10 & (IC95\%: 27,9-38,3) \\
\hline Risco & 66,90 & (IC95\%: 63,3-70,5) \\
\hline
\end{tabular}

IC: Intervalo de Confiança; M: Média; DP: Desvio-Padrão.

Observou-se que o consumo diário de frutas (PA:76,4\%, vs. A:93,1\%, $p=0,006$, respectivamente), leite e derivados (PA:76,4\% vs. A:93,2\%; $p=0,031)$ foi menor entre aqueles em pré-ação, sendo que usuários em ação relataram maior consumo de leite com baixo teor de gordura (PA: 19,6\% vs. A: 37,7\%, p=0,028) (Tabela 3).

Ao avaliar a ingestão de óleos e gorduras, verificou-se que os usuários classificados em pré-ação relataram consumo per capita mensal de óleo superior $(600,0 \mathrm{~mL}$ vs. $527,1 \mathrm{~mL} ; p=0,047)$, bem como ingestão diária de frituras, salgadinhos, carnes salgadas, hambúrgueres, presuntos e embutidos (PA:20,8\% vs. A:9,6\%; $p=0,013$ ) (Tabela 3). Em contraste, apresentaram menor frequência de consumo raro/nunca desses alimentos (PA:15,3\% vs. A:39,7\%; $p=0,013$ ) e relataram retirar sempre a gordura aparente da carne e a pele de frango (PA:63,9\% vs. A:89,0\%; $p<0,001$ ) (Tabela 3).

De acordo com a classificação do teste "Como está sua alimentação?", verificou-se que os usuários obtiveram uma média de 37,7, DP=6,0 pontos, 6,2\% precisavam tornar sua alimentação mais saudável, 71,7\% deveriam ficar atentos, e 22,1\% apresentavam alimentação saudável.

Não houve diferença da pontuação do teste segundo o comportamento alimentar (PA:35,94, $\mathrm{DP}=5,8$ vs. $\mathrm{A}: 39,54, \mathrm{DP}=5,7$ pontos; $p=0,993)$. Entretanto, os indivíduos em ação apresentaram uma melhor qualidade da dieta $(p=0,021)$, sendo essa diferença observada entre aqueles em pré-ação $(13,9 \%)$ e ação $(30,1 \%)$ que possuíam alimentação saudável $(p=0,034)$ (Figura 1). 
Tabela 2. Descrição e avaliação qualitativa do consumo de calorias e nutrientes segundo o comportamento alimentar dos usuários. Belo Horizonte (MG), 2008-2009.

\begin{tabular}{|c|c|c|c|c|c|}
\hline Variáveis & & Pré-ação & & Ação & Valor $p$ \\
\hline Calorias $(\mathrm{kcal})^{\mathbf{a}}$ & & $=1.882,2, \mathrm{DP}=542,4$ & & $=1.636,0, D P=548,0$ & $0,017^{*}$ \\
\hline Insuficiente (\%) & 66,7 & (IC95\% : 63,0-70,4) & 67,1 & (IC95\% : 63,5-70,7) & \\
\hline Adequado (\%) & 16,7 & $($ IC95\% : 12,1-21,3) & 17,8 & (IC95\% : 13,1-22,5) & $0,957^{* *}$ \\
\hline Excessivo (\%) & 16,7 & (IC95\% : 12,1-21,3) & 15,1 & (IC95\% : 10,7-19,5) & \\
\hline Carboidratos (\%) & & $M=49,5, D P=6,5$ & & $M=54,4, D P=6,7$ & $<0,001^{*}$ \\
\hline Insuficiente (\%) & 19,4 & $($ IC95\% : 14,6-24,2) & 8,2 & $($ IC95\% : $4,7-11,7)$ & \\
\hline Adequado (\%) & 80,6 & (IC95\% : 78,3-82,9) & 87,7 & (IC95\% : 86,2-89,2) & $0,034^{* \star *}$ \\
\hline Excessivo (\%) & 0,0 & $($ IC95\% : 0,0-0,0) & 4,1 & (IC95\% : 1,5-6,7) & \\
\hline Proteínas (\%) & & $M=16,20, D P=3,5$ & & $M=16,0, D P=3,3$ & $0,223^{*}$ \\
\hline Insuficiente (\%) & 4,2 & (IC95\% : 1,6-6,8) & 0,0 & $($ IC95\% : 0,0-0,0) & \\
\hline Adequado (\%) & 95,8 & (IC95\% : 95,2-96,4) & 100,0 & (IC95\% : 100,0-100,0) & $0,120^{* * *}$ \\
\hline Lipídeos (\%) & & $M=34,7, D P=4,9$ & $M=29$, & $D P=5,4$ & $<0,001^{*}$ \\
\hline Insuficiente (\%) & 0,0 & $($ IC95\% : 0,0-0,0) & 2,7 & $($ IC95\% : 0,5-4,8) & \\
\hline Adequado (\%) & 66,7 & (IC95\% : 63,0-70,4) & 89,0 & (IC95\% : 87,6-90,4) & $<0,001^{\star \star *}$ \\
\hline Excessivo (\%) & 33,3 & (IC95\% : 28,1-38,5) & 8,2 & $($ IC95\% : 4,7-11,7) & \\
\hline Ácido graxo saturado (\%) & 10,1 & (IC95\% : 6,2-13,9) & 8,1 & $($ IC95\% : 4,6-11,6) & $<0,001^{* * * *}$ \\
\hline Adequado (\%) & 52,8 & $($ IC95\% : 48,2-57,4) & 80,8 & (IC95\% : 78,5-83,1) & \\
\hline Excessivo (\%) & 47,2 & (IC95\% : 42,3-52,1) & 19,2 & (IC95\% : 14,4-23,9) & $<0,001^{* *}$ \\
\hline Ácido graxo monoinsaturado(\%)a & & $M=9,4, D P=2,2$ & & $M=7,7, D P=1,7$ & $<0,001^{*}$ \\
\hline Adequado (\%) & 90,3 & $($ IC95\% : 89,1-91,5) & 91,8 & (IC95\% : 90,7-92,8) & \\
\hline Excessivo (\%) & 9,7 & (IC95\% : 5,9-13,5) & 8,2 & (IC95\% : 4,7-11,7) & $0,751^{* *}$ \\
\hline Ácidos graxo poliinsaturado (\%) & & $M=9,3, D P=2,4$ & $M=9,0$ & $D P=2,6$ & $0,142^{*}$ \\
\hline Insuficiente (\%) & 8,3 & (IC95\% : 4,7-11,8) & 6,8 & $($ IC95\% : $3,5-28,9)$ & \\
\hline Adequado (\%) & 47,2 & (IC95\% : 42,3-52,1) & 67,1 & (IC95\% : 63,5-80,3) & \\
\hline Excessivo (\%) & 44,4 & $($ IC95\% : 39,4-49,3) & 26,0 & $($ IC95\% : 20,9-31,1) & $0,047^{* *}$ \\
\hline Colesterol $(\mathrm{mg})^{\mathbf{b}}$ & 200,8 & $($ IC95\% : 206,8-288,4) & 136,6 & (IC95\% : 153,8-210,4) & $0,004^{* * *}$ \\
\hline Adequado (\%) & 76,4 & (IC95\% : 73,6-79,2) & 86,3 & $($ IC95\% : 84,6-88,0) & \\
\hline Excessivo (\%) & 23,6 & $($ IC95\% : 18,6-28,6) & 13,7 & $($ IC95\% : 9,4-18,0) & $0,125^{* *}$ \\
\hline
\end{tabular}

a Média (M) e Desvio-Padrão (DP); ${ }^{\text {b }}$ Mediana e Intervalo de Confiança; ${ }^{*}$ Teste $t$ Student; ${ }^{* *}$ Teste Qui-quadrado de Pearson; ${ }^{* * *}$ Teste Exato de Fisher; **** Teste Mann-Whitney; IC: Intervalo de Confiança.

\section{DISCUSSÃ O}

O estudo apresentou elevada prevalência de indivíduos em estágios de pré-ação, segundo o Modelo Transteórico, que deviam ficar atentos com a alimentação de acordo com o teste utilizado. Esses dados corroboram os resultados identificados relativos ao estado nutricional e o consumo alimentar.

O perfil alimentar identificado se caracterizou pela ingestão reduzida de cereais e tubérculos, frutas, verduras e legumes, leite e derivados, e peixes; assim como pelo consumo excessivo de frituras, salgadinhos, carnes salgadas, hambúrgueres, presuntos e embutidos, e doces, biscoitos doces, bolo com cobertura e recheio, refrigerantes e sucos industrializados, alimentos ricos em sódio e com alta densidade energética.

Esses dados corroboram as mudanças ocorridas no padrão alimentar da população brasileira reveladas pela comparação entre o Estudo Nacional de Despesa Familiar (1974/1975) e a POF (2002/2003), que mostrou a redução do consumo de raízes e tubérculos (30\%), peixes (50\%), gordura animal (65\%); e o aumento da ingestão de biscoitos (400\%), embutidos (300\%), óleos e gorduras vegetais $(16 \%)^{18}$.

O consumo de carnes com excesso de gordura e pele de frango identificado neste estudo, tanto para indivíduos em pré-ação quanto em ação, foi abaixo do encontrado para a população brasileira $(34,6 \%)$, porém superior entre os pri- 
Tabela 3. Hábitos alimentares segundo o comportamento alimentar dos usuários. Belo Horizonte (MG), 2008-2009.

\begin{tabular}{|c|c|c|c|}
\hline Variáveis & Pré-ação & Ação & Valor $p$ \\
\hline Per capita mensal de óleo $(m L)^{\star \S}$ & 600,0 (IC95\%: 603,4-12,9) & 527,1 (IC95\%: 488,4-616,7) & 0,047 \\
\hline \multicolumn{4}{|l|}{ Tipo de gordura mais utilizado para cozinhar $(\%)^{\star *}$} \\
\hline Banha animal ou manteiga & 5,6 (IC95\%: 2,6-8,6) & 0,0 (IC95\%: 0,0-0,0) & \multirow[t]{2}{*}{0,058} \\
\hline Gordura vegetal & 94,4 (IC95\%: 93,7-95,1) & 100,0 (IC95\%: 100,0-100,0) & \\
\hline \multicolumn{4}{|l|}{ Tipo de leite e derivados que habitualmente consome $(\%)^{* *}$} \\
\hline Integral & 80,4 (IC95\%: 78,0-82,6) & 62,3 (IC95\%: 58,3-66,3) & \multirow[t]{2}{*}{0,028} \\
\hline Baixo teor de gordura & 19,6 (IC95\%: 14,8-24,4) & 37,7 (IC95\%: 32,6-42,8) & \\
\hline $\begin{array}{l}\text { Costuma retirar gordura aparente das carnes e pele do frango } \\
(\%)^{\star \star}\end{array}$ & 63,9 (IC95\%: 60,0-67,8) & 89,0 (IC95\%: 87,6-90,4) & $<0,001$ \\
\hline \multicolumn{4}{|l|}{$\begin{array}{l}\text { Frequência de consumo de frituras, salgadinhos, carnes salga- } \\
\text { das, hambúrgueres, presuntos, embutidos }(\%)^{* *}\end{array}$} \\
\hline Raramente ou nunca & 15,3 (IC95\%: 10,8-19,8) & 39,7 (IC95\%: 34,6-44,8) & \multirow[t]{5}{*}{0,013} \\
\hline 2 vezes por semana & 33,3 (IC95\%: 28,1-38,5) & 26,0 (IC95\%: 20,9-31,1) & \\
\hline 2 a 3 vezes por semana & 30,6 (IC95\%: 25,4-35,8) & 24,7 (IC95\%: 19,7-29,7) & \\
\hline 4 a 5 vezes por semana & 12,5 (IC95\%: 8,3-16,7) & $4,1(\mathrm{IC} 95 \%: 1,5-6,7)$ & \\
\hline Todos os dias & 8,3 (IC 95\%: 4,8-11,8) & $5,5(I C 95 \%: 2,5-8,5)$ & \\
\hline $\begin{array}{l}\text { Consumo diário de alimentos fonte de cereais e tubérculos } \\
\text { (porções) }^{\star}\end{array}$ & 4,0 (IC95\%: 3,9-4,7) & $3,7(I C 95 \%: 3,6-4,3)$ & 0,751 \\
\hline $\begin{array}{l}\text { Coloca mais sal quando os alimentos já estão servidos no pra- } \\
\text { to }(\%)^{\star *}\end{array}$ & 8,3 (IC95\%: 4,8-11,8) & 8,2 (IC95\%: 4,7-11,7) & 0,980 \\
\hline \multicolumn{4}{|l|}{ Número de refeições por dia $(\%)^{* *}$} \\
\hline 1 a 4 & 54,2 (IC95\%: 49,7-58,7) & 38,4 (IC95\%: 33,3-43,5) & \multirow[t]{2}{*}{0,056} \\
\hline 5 a 6 & 45,8 (IC95\%: 40,8-50,7) & 61,6 (IC95\%: 57,6-65,6) & \\
\hline \multicolumn{4}{|l|}{ Consumo diário de carnes (de qualquer tipo) e ovos (\%) } \\
\hline Não consome & 2,8 (IC95\%: 0,6-5,0) & 6,8 (IC95\%: 3,5-10,1) & \multirow{4}{*}{0,610} \\
\hline 1 pedaço/fatia/colher de sopa/ovo & 19,4 (IC95\%: 14,6-24,2) & 23,3 (IC95\%: 18,3-28,3) & \\
\hline 2 pedaços/fatias/colheres de sopa/ovos & 37,5 (IC95\%: 32,4-42,6) & 35,6 (IC95\%: 30,4-40,8) & \\
\hline Mais de 2 pedaços/fatias/colheres de sopa/ovos & 40,3 (IC95\%: 35,2-45,4) & 34,2 (IC95\%: 29,0-39,4) & \\
\hline \multicolumn{4}{|l|}{ Frequência de consumo de peixe (\%) } \\
\hline Não consome & 2,8 (IC95\%: 0,6-5,0) & 8,2 (IC95\%: 4,7-11,7) & \multirow{4}{*}{0,581} \\
\hline Algumas vezes no ano & 41,7 (IC95\%: 36,6-46,8) & 39,7 (IC95\%: 34,6-44,8) & \\
\hline 1 a 4 vezes por mês & 4,2 (IC95\%: 1,6-6,8) & 4,1 (IC95\%: 1,5-6,7) & \\
\hline 2 ou mais vezes por semana & 51,4 (IC95\%: 46,7-56,1) & 47,9 (IC95\%: 43,1-52,7) & \\
\hline \multicolumn{4}{|l|}{ Consumo diário de leite e derivados (\%) } \\
\hline Não consome & 23,6 (IC95\%: 18,6-28,6) & 6,8 (IC95\%: 3,5-10,1) & \multirow{4}{*}{0,031} \\
\hline 1 ou menos copos ou pedaços/fatias/porções & 20,8 (IC95\%: 15,9-25,7) & 32,9 (IC95\%: 27,7-38,1) & \\
\hline 2 copos ou pedaços/fatias/porções & 15,3 (IC95\%: 10,8-19,8) & 15,1 (IC95\%: 10,7-19,5) & \\
\hline 3 ou mais copos ou pedaços/fatias/porções & 40,3 (IC95\%: 35,2-45,4) & 45,2 (IC95\%: 40,3-50,1) & \\
\hline \multicolumn{4}{|l|}{ Consumo diário de legumes e verduras (\%) } \\
\hline Não consome & 1,4 (IC95\%: -0,2-3,0) & 0,0 (IC95\%: 0,0-0,0) & \multirow{5}{*}{0,241} \\
\hline 3 ou menos colheres de sopa & 31,9 (IC95\%: 26,7-37,1) & 21,9 (IC95\%: 17,0-26,8) & \\
\hline 4 a 5 colheres de sopa & 29,2 (IC95\%: 24,1-34,3) & 23,3 (IC95\%: 18,3-28,3) & \\
\hline 6 a 7 colheres de sopa & 18,1 (IC95\%: 13,4-22,8) & 24,7 (IC95\%: 19,7-29,7) & \\
\hline 8 ou mais colheres de sopa & 19,4 (IC95\%: 14,6-24,2) & 30,1 (IC95\%: 24,9-35,3) & \\
\hline
\end{tabular}

IC: Intervalo de Confiança; *Mann-Whitney; ${ }^{* *}$ Teste Qui-quadrado de Pearson; §Estimativa realizada por meio das questões: "Quantos frascos de óleo você utiliza por mês?" e "Quantas pessoas utilizam o óleo consumido no mês?".

Nota: Teste Qui-quadrado de Pearson. 
Tabela 3. Hábitos alimentares segundo o comportamento alimentar dos usuários. Belo Horizonte (MG), 2008-2009.

\begin{tabular}{|c|c|c|c|c|c|}
\hline Variáveis & & Pré-ação & & Ação & Valor $p$ \\
\hline \multicolumn{6}{|l|}{ Consumo diário de frutas (\%) } \\
\hline Não consome & 23,6 & $($ IC95\% : 18,6-28,6) & 6,8 & $($ IC95\% : 3,5-10,1) & \multirow{4}{*}{0,006} \\
\hline 1 unidade ou porção & 29,2 & (IC95\% : 24,1-34,3) & 53,4 & (IC95\% : 48,8-58,0) & \\
\hline 2 unidades ou porção & 33,3 & $(I C 95 \%: 28,1-38,5)$ & 30,1 & $($ IC95\% : 24,9-35,3) & \\
\hline 3 ou mais unidades ou porção & 13,9 & $($ IC95\% : 9,6-18,2) & 9,6 & $($ IC95\% : 5,8-13,4) & \\
\hline \multicolumn{6}{|c|}{$\begin{array}{l}\text { Frequência de consumo de doces, biscoitos doces, bolos re- } \\
\text { cheados com cobertura, refrigerantes, sucos industrializados } \\
\text { (\%) }\end{array}$} \\
\hline Raramente ou nunca & 16,7 & (IC95\% : 12,1-21,3) & 27,4 & $(I C 95 \%: 22,3-32,5)$ & \multirow{5}{*}{0,245} \\
\hline 2 vezes por semana & 20,8 & (IC95\% : 15,9-25,7) & 21,9 & (IC95\% : 17,0-26,8) & \\
\hline 2 a 3 vezes por semana & 30,6 & $($ IC95\% : 25,4-35,8) & 17,8 & $($ IC95\% : 13,1-22,5) & \\
\hline 4 a 5 vezes por semana & 12,5 & $($ IC95\% : 8,3-16,7) & 8,2 & $($ IC95\% : 4,7-11,7) & \\
\hline Todos os dias & 19,4 & (IC95\% : 14,6-24,2) & 24,7 & $($ IC95\% : 19,7-29,7) & \\
\hline
\end{tabular}

Nota: Teste Qui-quadrado de Pearson.

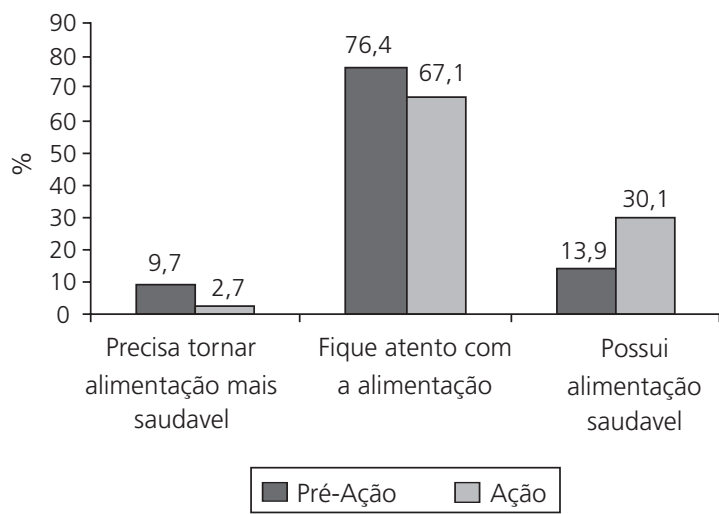

Figura 1. Comparação do escore do Teste "Como está sua alimentação?" e da classificação dos estágios de mudança de comportamento alimentar para consumo de óleos e gorduras dos usuários. Belo Horizonte (MG), 2008-2009.

Nota: Prevalências e Intervalos de Confiança 95\%: 9,7\% (5,9-13,5); $2,7 \%(0,6-4,8) ; 76,4 \%(73,6-79,2) ; 67,1 \%(63,5-70,7) ; 13,9 \%$ $(9,6-18,2) ; 30,1 \%(24,9-35,2)$. Teste Exato de Fisher $(p=0,021)$; * Teste Qui-quadrado de Pearson $(p=0,034)$

meiros, como esperado, enquanto a ingestão de leite e derivados com teor integral de gordura foi superior à de adultos brasileiros (56,9\%), sendo maior entre indivíduos em pré-ação ${ }^{4}$. Destaca-se que o consumo per capita mensal de óleo de ambos os grupos foi bastante superior à recomendação do Ministério da Saúde ${ }^{5,6}$.
Apesar do elevado consumo de óleo e de alimentos gordurosos, identificou-se que a distribuição média de macronutrientes e a ingestão média de Ácido Graxo Monoinsaturado (AGM), AGP e colesterol em indivíduos em pré-ação e ação atendiam às necessidades recomendadas, estando somente a ingestão de AGS acima dos valores preconizados ${ }^{16,17}$.

Na avaliação qualitativa do consumo, notou-se elevada prevalência de consumo insuficiente de calorias em ambos os grupos. Por outro lado, as prevalências de ingestão excessiva de lipídeos, AGS, AGP e colesterol foram maiores naqueles em pré-ação, enquanto a ingestão de carboidratos, proteínas e AGM foi superior entre indivíduos em ação, e a média de consumo energético inferior.

Ao comparar os percentuais médios de consumo obtidos neste estudo com os valores oriundos da POF (2002/2003), verificou-se que foram semelhantes para todos os nutrientes analisados (carboidratos: 55,9\%, proteínas: 13,6\%, lipídeos: 30,5\%, AGS: 9,6\%, AGM: 8,1\%, AGP: $8,9 \%)^{6,18}$.

No estudo de Gorbach et al. ${ }^{19}$, as taxas de ingestão de lipídeos (39,0\%), AGS (13,9\%), AGM $(13,9 \%)$ e colesterol (310mg) foram superiores 
às encontradas neste estudo, com proximidade somente para AGP (7,3\%). No trabalho de Nasser et al. ${ }^{20}$, observou-se que as taxas de consumo de lipídeos (30\%), AGS (9\%) e AGP (6\%) foram próximas às encontradas, apresentando-se superior somente para AGM (11\%).

O consumo excessivo de lipídeos, AGS e colesterol encontrado neste estudo pode ser explicado pelo alto consumo de produtos industrializados, com elevada densidade calórica, e de alimentos de origem animal, ricos em gorduras saturadas e colesterol ${ }^{5,18,21}$. Esse dado é preocupante uma vez que a ingestão de alimentos ou preparações ricas em colesterol e AGS periodicamente e em quantidades excessivas aumentam o risco de DANT, como doenças cardiovasculares, cânceres e diabetes, por meio de seus efeitos nas concentrações de lipoproteínas ${ }^{22}$; assim como o consumo em excesso de AGP pode contribuir para o desenvolvimento de obesidade, doenças inflamatórias e cardiovasculares ${ }^{23}$.

A alta prevalência de consumo insuficiente de calorias, por sua vez, pode ser explicada pelo sub-relato do consumo, provavelmente devido à porcentagem elevada de indivíduos obesos ou pré-obesos e mulheres ${ }^{24}$ que podem apresentar percepção errônea de seu consumo alimentar ${ }^{8,25}$. Além disso, pode haver a subestimação oriunda da dificuldade em se quantificarem os nutrientes por meio de tabelas de composição, sendo por isso utilizadas neste estudo diferentes tabelas de composição de alimentos, bem como rótulos de produtos e receitas, quando necessário.

$\mathrm{Na}$ avaliação da qualidade da dieta, a maioria dos indivíduos apresentou-se no escore "deve ficar atento com a alimentação", o que condiz com o perfil alimentar identificado. Esse dado também demonstra similaridade com a avaliação do comportamento alimentar para o consumo de óleos e gorduras, na qual foi detectada elevada prevalência de indivíduos em pré-ação que apresentavam consumo excessivo de alimentos fontes de lipídeos. Por sua vez, a alimentação considerada saudável foi mais prevalente entre os indivíduos em ação, corroborando as caracte- rísticas dos próprios estágios de ação e manutenção ${ }^{25}$. Tais dados sugerem a aplicabilidade do teste como instrumento que contribui para o conhecimento da qualidade da alimentação de indivíduos e grupos populacionais.

Ao comparar os escores da avaliação da qualidade da dieta com os estágios de mudança de comportamento alimentar para consumo de óleos e gorduras, verificou-se que a maioria dos usuários tanto em pré-ação quanto em ação deveriam ficar atentos com sua alimentação. Esse dado sugere que os indivíduos em ação, apesar de já realizarem modificações em seus hábitos alimentares, ainda necessitam concretizar mais mudanças em seu comportamento alimentar, o que caracteriza o dinamismo dos estágios, com constantes hábitos a serem modificados e repensados $^{7}$. Assim, o sucesso de intervenções que visam a tais mudanças também depende da capacidade do indivíduo em aprender novas competências, de reajustar seu estilo de vida e da capacidade para lidar com a fadiga ${ }^{26}$, promovendo outras mudanças necessárias.

Entretanto, como o escore de qualidade da dieta se propõe a realizar uma avaliação global da dieta e do algoritmo utilizado e identificar o comportamento alimentar para o consumo de óleos e gorduras, verifica-se a necessidade de se avaliar o comportamento para outros grupos alimentares, visando a uma avaliação mais ampla.

Além disso, são escassos os estudos que utilizam o Modelo Transteórico no Brasil; para o consumo de óleos e gorduras, os estudos existentes foram realizados em outros países, com populações de níveis socioeconômico e educacional distintos do grupo estudado, e com diferentes algoritmos e métodos de avaliação, dificultando a comparação com a literatura. Ressalta-se que Hart et al. ${ }^{27}$ demonstrou em seu estudo que idade, raça, educação, sexo e saúde apresentam correlação significativa com a ingestão de gordura.

Quanto ao uso de diferentes algoritmos e métodos de reclassificação, notou-se que somente o estudo realizado por Greene \& Rossi ${ }^{8}$ utilizou 
método semelhante para a reclassificação dos estágios de mudança, porém os valores diferiram (Pré-Ação: 80,0\% e Ação: 13,0\%). Lechener et al.22, apesar de usar como instrumento de avaliação de consumo de óleos e gorduras o Questionário de Frequência Alimentar, obteve resultados semelhantes ao encontrado (Pré-Ação: 83,0\% e ação: 17,0\%). Esses dados também foram observados nos estudos de Auld ${ }^{28}$ (Pré-Ação: 56,0\% e ação: 45,0\%); e de Plotonikoff21 (Pré-Ação: 22,4\% e ação: $77,6 \%$ ).

O uso de métodos de avaliação do comportamento alimentar e da qualidade da dieta conjuntamente é relevante, uma vez que propicia avaliar a dieta de forma qualitativa e quantitativa, além de possibilitar a análise dos determinantes do comportamento alimentar e compreender as mudanças dos hábitos alimentares.

Ademais, a avaliação da qualidade da dieta pelo instrumento proposto pelo Ministério da Saúde parece ser uma alternativa viável a ser utilizada em serviços de saúde, uma vez que constitui um instrumento simples e de rápida aplicação, além de ser útil para a descrição e o monitoramento do padrão alimentar, de permitir avaliar intervenções e apresentar resultados similares a método já validado, como o algoritmo aqui utilizado.

\section{O N C L U S Ã O}

Ao utilizar o Modelo Transteórico para avaliar o comportamento alimentar, detectou-se elevada prevalência de indivíduos nos estágios iniciais para consumo de óleos e gorduras, ou seja, em pré-ação. Demonstrou-se ainda que o comportamento alimentar obtido por esse método é condizente com a qualidade da dieta avaliada pelo teste "Como está sua alimentação?", uma vez que aqueles em pré-ação apresentaram pior qualidade da dieta, caracterizada por menor consumo de frutas, leites e derivados; e maior consumo de óleos e alimentos ricos em gorduras. Entretanto, no grupo ação identificou-se maior proporção de indivíduos classificados como possuindo uma alimentação saudável, segundo o escore do teste, apresentando hábitos mais saudáveis como consumir leite com baixo teor de gordura e retirar sempre a gordura aparente da carne e a pele de frango.

Diante da similaridade dos dados obtidos por ambos os métodos, evidencia-se a necessidade de avaliar os hábitos alimentares e o comportamento alimentar da população, visando identificar as necessidades de mudanças dos hábitos alimentares, e melhor planejar, implementar e avaliar intervenções, de acordo com os estágios de mudança comportamental.

Ademais, o uso conjunto de questionários relativos à avaliação de comportamento e hábitos alimentares denota a importância de se compreederem as diferenças de consumo de alimentos conforme a mudança comportamental dos indivíduos.

\section{A GRADECIMENTOS}

À Fundação de Apoio à Pesquisa do Estado de Minas Gerais, pelo financiamento do projeto, e à Coordenaação de Aperfeiçoamento de Pessoal de Nível Superior, pela bolsa de mestrado. À equipe de pesquisa, em especial à Cristilene, à Raquel Mendonça e à Vanessa; e à equipe do serviço, Danielle, Thiago, André, Rafael, Richardison e Talita.

\section{REFERÊ NCIAS}

1. Organização Mundial de Saúde. Prevenção de doenças crônicas: um investimento vital. Genebra: OMS; 2005 [acesso 2008 out 03]. Disponível em: $<$ http://www.who.int/chronic_disease_report/en/>.

2. Instituto Brasileiro de Geografia e Estatística. Pesquisa de Orçamentos Familiares 2008/2009: aquisição alimentar domiciliar per capita, Brasil e grandes Regiões. Brasília: IBGE; 2010.

3. World Health Organization. Nutrition: controlling the global obesity epidemic. Geneva: WHO; 2002 [cited 2008 Oct 3]. Available from: <http://www. who.int/nut/obs.htm>.

4. Brasil. Ministério da Saúde. Vigilância de fatores de risco e proteção para doenças crônicas por inquérito telefônico: 2011. Brasília: MS; 2012.

5. Brasil. Ministério da Saúde. Guia alimentar para a população brasileira: promovendo a alimentação saudável. Brasília: MS; 2006. 
6. Brasil. Ministério da Saúde. Guia Alimentar de Bolso como ter uma alimentação saudável: dez passos para uma alimentação saudável. Brasília: MS; 2007.

7. Prochaska JO, Redding CA, Evers KE. The Transtheoretical Model and stages of change. In: Glanz K, Lewis FM, Rimer BK, editors. Health behavior and health education: theory, research, and practice. $2^{\text {nd }}$ ed. California: Jossey-Bass; 1996.

8. Greene GW, Rossi SR. Stages of change reducing dietary fat intake over 18 months. J Am Diet Assoc. 1998; 18(5):529-34. doi: 10.1016/S0002-8223 (98)00120-5.

9. Jomori MM, Proença RPC, Calvo MCM. Determinantes de escolha alimentar. Rev Nutr. 2008; 21(1):63-73. doi: 10.1590/S1415-5273200800010 000.

10. Dias MAS, Giatti L, Guimarães VR, Amorim MA, Rodrigues CS, Lansky S, et al. BH-Saúde: projeto promoção de modos de vida saudáveis. Pensar BH/ Política Social. 2006; 16:21-4. doi: 10.1590/S141552732008000100007.

11. Moreira RAM. Aplicação do modelo transteórico para consumo de óleos e gorduras e sua relação com o consumo alimentar e estado nutricional em um Serviço de Promoção da Saúde [mestrado]. Belo Horizonte: Universidade Federal de Minas Gerais; 2010.

12. World Health Organization. Physical status: the use and interpretation of anthropometry. Geneva: WHO; 1995. Technical Report Series 854.

13. Nutrition Screening Initiative. Nutrition interventions manual for professionals caring for older Americans. Washington: NSI; 1992.

14. World Health Organization. Burden: mortality, morbidity and risk factors. In: Global status report on noncommunicable diseases 2010. Geneva: WHO; 2011.

15. Fisberg RM, Martini LA, Slater B. Métodos de inquéritos alimentares. In: Fisberg RM, Slater B, Marchioni DML, Martini LA. Inquéritos alimentares: métodos e bases científicos. Barueri: Manole; 2005.

16. Institute of Medicine. Dietary reference intakes for energy, carbohydrate, fiber, fat, fatty acids, cholesterol, protein, and amino acids (macronutrients). Washington: The National Academies Press; 2005 [cited 2008 Sept 20]. Available from: <http://www.nal.usda.gov/fnic/DRI/ /DRI_Energy/energy_full_report.pdf>.

17. World Health Organization. Diet, nutrition and the prevention of chronic diseases. Geneva: WHO: 2003. Technical Report Series, 916.

18. Levy-Costa RB, Sichieri R, Pontes NS, Monteiro CA. Disponibilidade domiciliar de alimentos no Brasil: distribuição e evolução (1974-2003). Rev Saúde Pública. 2005, 39(4):530-40. doi: 10.1590/S003489102005000400003.
19. Gorbach SL, Morril-LaBrode A, Woods MN, Dwyer JT, Selles WD, Henderson M, et al. Changes in food patterns during a low-fat dietary intervention in women. J Am Diet Assoc. 1990, 90(6):802-9. doi: 10.1001/archinte.1990.00390140121026.

20. Nasser R, Cook SL, Dorsch HD, Haennel RG. Comparison of two nutrition education approaches to reduce dietary fat intake and serum lipids reveals registered dietitians are effective at disseminating information regardless of the educational approach. J Am Diet Assoc. 2006, 106(6):850-9. doi: 10.1016/j.jada.2006.03.011.

21. Plotinikoff RC, Lippke S, Johnson ST, Hotz SB, Birkett NJ, Rossi SR. Applying the stages of changeto multiple low-fat dietary behavioral contexts: an examination of stage occupation and discontinuity. Appetite. 2009, 53(3):345-53. doi: 10.1016/j.appet.2009.07.016.

22. Lechner L, Brug J, De Vries H, Assema PV, Mudde A. Stages of change for fruit, vegetable and fat intake: consequences of misconception. Health Educ Res. 1998, 13(1):1-11. doi: 10.1093/her/ 13.1.1.

23. Martin CA, Almeida W, Ruiz MR, Visentainer JEL, Matshushita M, Souza NE, et al. Ácidos graxos poliinsaturados ômega-3 e ômega-6: importância e ocorrência em alimentos. Rev Nutr. 2006, 19(6): 761-70. doi: 10.1590/S1415-52732006000600 011.

24. Scagliusi FB, Lancha Júnior AH. Subnotificação da ingestão energética na avaliação do consumo alimentar. Rev Nutr. 2003, 16(4):471-81. doi: 10.15 90/S1415-52732003000400010.

25. Prochaska JO, Johnson S, Lee P. The transtheoretical model of behavior change. In: Shumaker S, Schron E, Ockene J, Mcbee W, editors. The handbook of health behavior change. $2^{\text {nd }} \mathrm{ed}$. New York: Springer Publishing Company; 1998.

26. De Graff C, Van der Gaag M, Kafatos A, Lennernas $M$, Kearney JM. Stages of dietary change among nationally-representative samples of adults in the European Union. Eur J Clin Nutr. 1997, 51(2): S47-S56.

27. Hart Jr A, Tinker L, Bowen DJ, Longton G, Beresfor SA. Correlates of fat intake behaviors in participants in the eating for a health life study. J Am Diet Assoc. 2006, 106(10):1605-13. doi:10.1016/j.jada.2006.0 7.006.

28. Auld GW, Nitzke SA, McNulty J, Bock MA, Bruhn CM, Gabel K, et al. A stage-of-change classification system based on actions and beliefs regarding dietary fat and fiber. Am J Health Promot. 1998, 12(3):192-201. doi: 10.4278/0890-1171-12.3.192.

Recebido em: 24/2/2012

Versão final em: 13/7/2012

Aprovado em: 6/9/2012 